\title{
Perceptual cues for sentence intonation in Greek
}

Anthi Chaida

Phonetics Laboratory, University of Athens, Greece

https://doi.org/10.36505/ExLing-2010/03/0006/000126

\begin{abstract}
This is a study of intonational cues for the perception of statements and polar questions in Greek. A two-part experiment was conducted with synthetic stimuli with manipulated intonation targets in stress groups, from statement to question and vice versa. According to the results, the critical perceptual cue is the boundary tone, which led listeners to identify stimuli as the reverse sentence type (81-100\%). However, the perception of intonational contrasts may be based on holistic contours.
\end{abstract}

Key words: perception, intonation, prosody, sentence types, Greek.

\section{Introduction}

The present study investigates the effects of prosody on sentence perception in Greek, i.e. the decisive perceptual cues for statements and polar questions, based on synthetic stimuli, on which the intonational contours of statements are partly and totally replaced by question appropriate contours, and vice-versa. In previous studies natural stimuli of all types were perceived with $99 \%$ accuracy. In general, intonation has been shown to contribute to the production and perception of sentence type contrasts in many languages (e.g. Chaida 2008, Makarova 2001, Thorsen, 1980).

\section{Materials and method}

A set of utterances produced by one female speaker at her twenties was selected from a previously recorded corpus (Chaida 2007), including the sentence [o ma'nolis ma'zevi lemona] (Manolis is picking lemons), produced as statement and polar question. These utterances were processed with Praat. Their pitch was stylized (frequency resolution 1 semitone), and they were manipulated in two ways: (a) the 3 stress groups of the statement were manipulated, one by one, in pairs, and in total, in order to reach the pitch targets of the question (with the same alignment), but maintaining duration and intensity, and vice versa (examples in Fig. 1-2.3); (b) the 3 stress groups of the statement where replaced unedited (carrying duration and intensity), one by one, by the corresponding stress groups of the question, and vice-versa. A set of 14 stimuli was set up by the 1st procedure and a set of 6 synthetic stimuli by the 2 nd. The stylized original utterances of a statement and a polar question were included in both sets. All stimuli were randomized 4 times. These modified stimuli were presented to 30 informants (male-female, 20-40 years

ExLing 2010: Proceedings of 3rd Tutorial and Research Workshop on Experimental Linguistics, 25-27 August, Athens, Greece 
old from Athens). The stimuli were presented over headphones in a quiet room, and the informants were instructed to identify each stimulus as either a statement or a question, and then to provide an indication of how certain they were of their decision (1-6 scale: $1=$ least, $6=$ most certain).

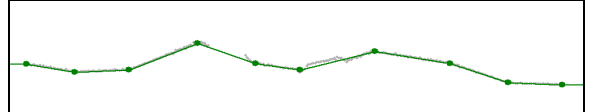

Figure 1. Intonation contour for statement with stylized pitch.

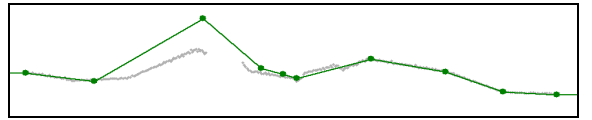

Figure 1.1. Statement with question's tonal onset (1st stress group).

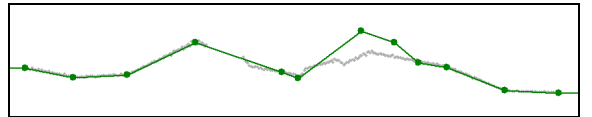

Figure 1.2. Statement with question's medial (2nd) stress group.

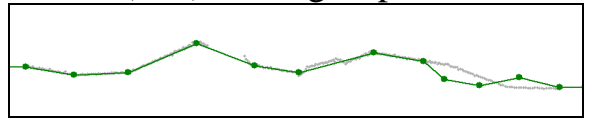

Figure 1.3. Statement with question's tonal offset (3rd stress group).

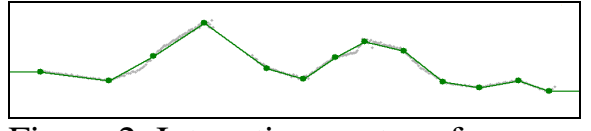

Figure 2. Intonation contour for polar question with stylized pitch.

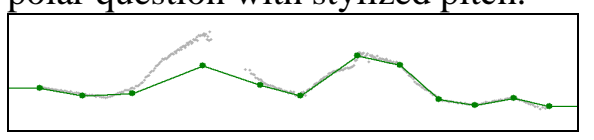

Figure 2.1. Question with statement's tonal onset (1st stress group).

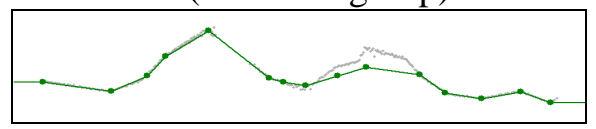

Figure 2.2. Question with statement's medial (2nd) stress group.

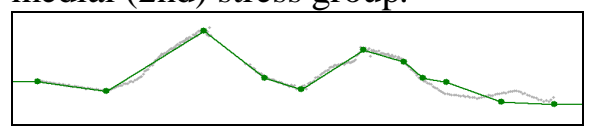

Figure 2.3. Question with statement's tonal offset (3rdt stress group).

\section{Results}

The original stylized stimuli had very high correct identification rates in total: $98.35 \%$ for statements and $99.57 \%$ for questions. According to the results of the 1st part of the experiment, statements had high correct identification scores (perceived as statements) for stimuli with manipulation on the 1st stress group (99.16\%), on the 1st and 2nd (100\%), and on the 2nd one $(96.66 \%)$. However, when the boundary tone was manipulated, statements were identified as questions: $80 \%$ for the 1st and 3rd stress group, $81.66 \%$ for the 2 nd and $3 \mathrm{rd}$, and $81.66 \%$ for the $3 \mathrm{rd}$ stress group. In the cases where the intonation contour of the statement had been totally replaced by the question, listeners mostly identified statements as questions (80\%). The results for questions were similar, with high correct identification scores for stimuli with manipulation on the 1st stress group (99.16\%), on the 1st and 2nd (99.16\%), and on the 2nd one (99.16\%). Here again, when listeners were presented stimuli with manipulation on the 3rd stress group (boundary), they identified questions as statements: $98.33 \%$ for the 1st and 3rd stress group, $98.33 \%$ for the 2 nd and 3 rd, and $100 \%$ for the 3rd stress group. In 
the cases where the intonation contour of the question had been totally replaced by the statement one, listeners mostly identified questions as statements $(99.16 \%)$.

Certainty ratings were fairly high: 5.21 (mean) out of 6 for statements, and 4.78 for questions. These ratings were statistically significant for questions $(F(1,872)=83.72, p<0.01$, $)$, but not for statements $(F(1,1044)=0.41, p>0.01$,$) .$ Listeners seemed to be most certain when they answered for stimuli with manipulations on the whole of the intonation contour (5.32 out of 6), and least certain for stimuli with combined manipulations on the 1 st and 3rd stress group (4.65).

According to the results of the 2nd part of the experiment (stimuli with replaced unedited prosodic parts), statements had high identification score (perceived as statements) in stimuli with manipulation on the 1st stress group (99.16\%), and on the 2nd one (100\%), while stimuli with the 3rd stress group replaced (boundary tone) were noticeably identified as questions (97.50\%). Questions also had high identification scores, i.e. they were perceived as questions with manipulation on the 1st stress group (100\%), and on the 2nd one (100\%), and as statements with manipulation on the 3rd stress group (100\%).

Certainty ratings were fairly high in general: 5.47 (mean) out of 6 for statements, and 5.38 for questions. These ratings were statistically significant for questions $(\mathrm{F}(1,474)=30.87, \mathrm{p}<0.01)$, but not for statements $(F(1,482)=8.73, p>0.01)$. Listeners seemed to be most certain when they responded to stimuli with manipulations on the 1st stress group (5.55 out of 6 ), and least certain for stimuli with manipulations on the 3rd stress group (5.16), but the range was very short in all cases.

In both parts of the experiment, the identification results were statistically significant (chi-square test, $\mathrm{p}<0.01$ ) and the listeners' choices were not incidental, since their answers were in accordance with the initial sentence types and stress group manipulations at the $99 \%$ confidence level.

\section{Discussion}

Several studies have shown that each sentence type has an acoustically distinct tonal structure, characterized in Greek by the type and location of the tonal nucleus and the boundary tone (Chaida 2005, 2007, Botinis Bannert, Tatham 2000). Moreover, experiments with artificial hum stimuli without any lexical information showed that prosody is a major cue for the perception of statements and questions in Greek (Chaida 2008).

The discrete intonational contours of sentence types are transparently distinguished by native speakers. There is a clear 1 to 1 production-perception correspondence. However, the crucial question of the present study is: what are the decisive intonational cues for the perceptual differentiation of statements and polar questions in Greek? 
differentiation of statements and polar questions in Greek? The results indicate that sentence type perception is mostly based on the realization of the boundary tone. In both parts of the experiment, the listeners identified statements as questions, and questions as statements. These findings agree in general with earlier studies demonstrating that in many languages low or falling boundary tones elicit 'declarative judgments', and high or rising tones lead to 'interrogative judgments' (e.g. Baltazani, 2007; Makarova, 2001; Thorsen, 1980). Moreover, it has been found that, although in general tonal cues for sentence types are distributed over the whole utterance, the last stressed syllable and the following unstressed ones are sufficient indicators for sentence type perception (Thorsen, 1980). It has been suggested that the entire intonation contour, rather than timing and scaling of turning points, can have impact on perception; i.e. perception of intonational contrasts is mostly based on holistic contours, rather than on local characteristics (Knight, 2008; Niebuhr, 2003). Hence, although the boundary tone (tonal offset) has been found to be a major perceptual cue for listeners, its effect may be overridden by earlier tonal movements, confirming the importance of the entire contour for perception.

\section{References}

Baltazani, M. 2007. Intonation of polar questions and the location of nuclear stress in Greek. In: Gussenhoven, C., Riad, T. (eds.), Tones and tunes-II. Experimental Studies in Word and Sentence Prosody. Berlin: Mouton de Gruyter, 387-405.

Botinis, A., Bannert, R., Tatham, M. 2000. Contrastive tonal analysis of focus perception in Greek and Swedish. In: Botinis, A. (ed), Intonation: Analysis, Modelling and Technology. Dordrecht: Kluwer Academic Publishers, 97-116.

Chaida, A. 2005. Intonation of sentence types and focus in Greek: production and perception. MA Thesis, University of Skövde, Sweden.

Chaida, A. 2007. Tonal structures of complex sentences in Greek. Proc. of the 8th International Conference on Greek Linguistics. Ioannina, Greece.

Chaida, A. 2008. Prosodic perception of sentence types in Greek. 2nd ISCA Workshop on Experimental Linguistics, Athens, Greece, 61-64.

Knight, R. 2008. The Shape of nuclear falls and their effect on the perception of pitch and prominence. Language and Speech, 51(3): 223 - 244.

Makarova, V. 2001. Perceptual correlates of sentence-type intonation in Russian and Japanese. Journal of Phonetics, 29, 137-154.

Niebuhr, O. 2003. Perceptual study of timing variables in F0 peaks. Proceedings of the 15th ICPhS, Barcelona, Spain, 1225-1228.

Thorsen N. 1980. A study of the perception of sentence intonation - evidence from Danish. Journal of the Acoustical Society of America, 67, 1014-1030. 